\title{
Numerical Method for the Kinematic Analysis of the Spatial Multi-Link Mechanisms
}

\author{
Cătălin Alexandru
}

\begin{abstract}
The work deals with a numerical method for the kinematic analysis of the spatial multi-link mechanical systems (linkages). According to the proposed method, three specific points determine the spatial position and orientation of the central element of the mechanism (i.e. the rod). The kinematic equations system contains the geometric constraint equations and the rigid body conditions of the rod (i.e. constant distances between the three specific points). The corresponding non-linear system is solved by using the Newton-Kantorovich approach. The case study is developed by considering a complex wheel guiding mechanism used for vehicle suspension system.
\end{abstract}

Index Terms-Multi-link mechanism, kinematic analysis, analytical algorithm, guiding linkage.

\section{INTRODUCTION}

The articulated mechanical systems (linkage mechanisms) are the most common types of mechanisms due to the multiple functions they can provide. There is practically no machine (mechanical, hydraulical, electrical, etc.) or equipment that does not integrate such mechanisms. As applications, the following can be pointed: working mechanisms, indicator mechanisms, lifting and transport mechanisms, actuators and controls, guiding mechanisms, etc., with various fields of use (automobiles, aircraft, fine mechanics, technological equipment, agricultural machinery, textile machinery, electrical devices etc.).

A general systematization of the linkage mechanisms, which responds to the main technical problems solved with them, can be structured is the following way (figure 1):

a. positioning (guiding) mechanisms, which perform a sequence of positions for a specific element of the mechanism, usually the central element - the rod (ex. dough kneading machine mechanism - a.1; forklift platform tipping mechanism - a.2; garage door moving mechanism - a.3);

b. trajectory generating mechanisms, which generate the prescribed trajectory by some specific points of the mechanism (ex. projector film drive mechanism - b.1; sewing machines thread pulling \& stretching mechanism - b.2; harbor crane mechanism - b.3);

c. functions generating mechanisms, which achieve a certain functional dependence between the driving and driven elements of the mechanism (ex. writing machine mechanism c. 1 ; logarithm function generating mechanism - c.2; weighing mechanism - c.3).

Manuscript received February, 2018; revised April 26, 2018.

C. Alexandru is with the Product Design, Mechatronics and Environment Department, Transilvania University of Braşov, 500036 Braşov, Romania (e-mail: calex@unitbv.ro).
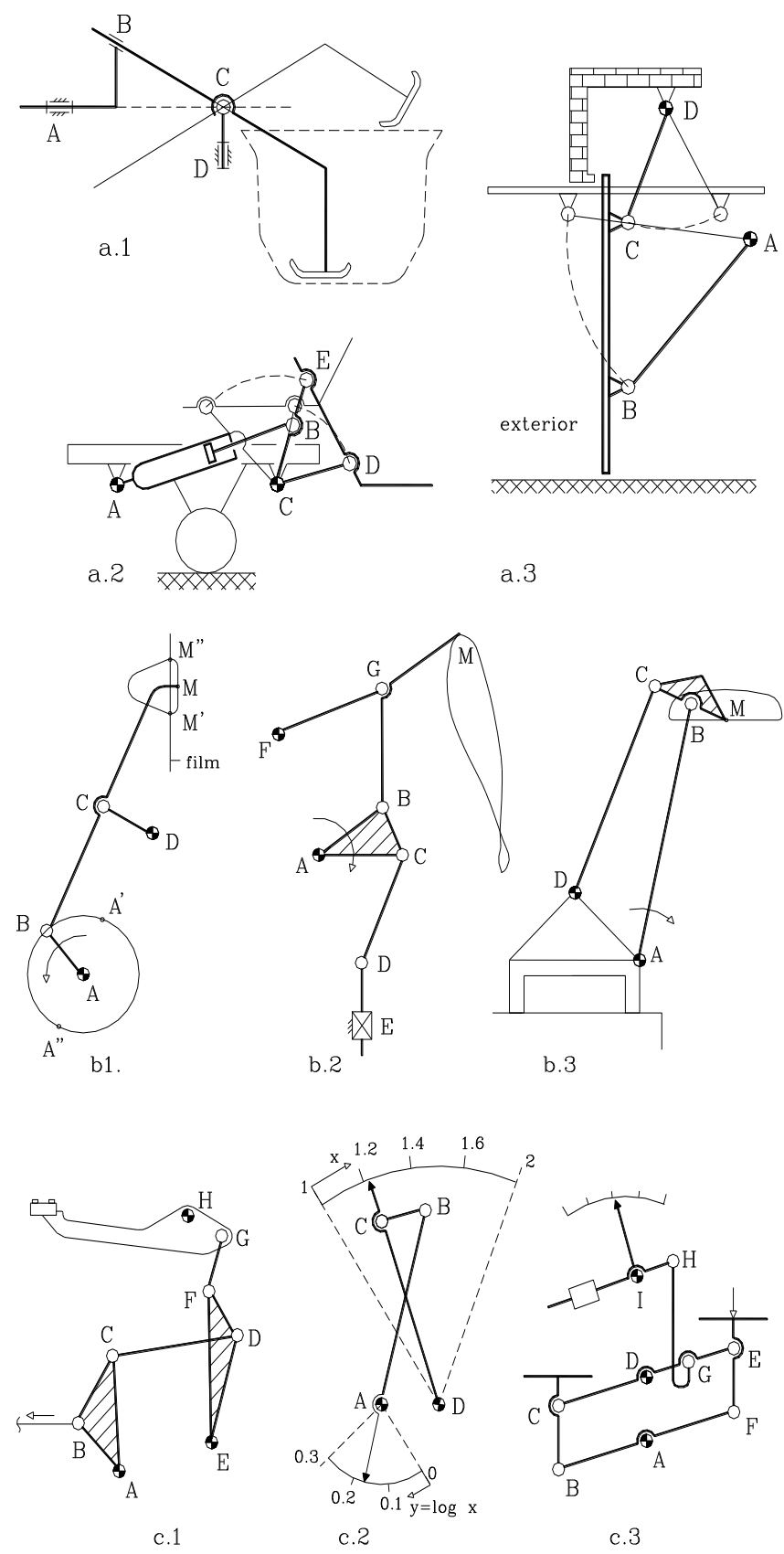

Fig. 1. Representative types of mechanisms.

The functional-kinematic design of the multi-link mechanisms usually involves the following steps:

- establishing the concrete functional requirements that the mechanism must satisfy;

- defining the geometric-kinematic model of the mechanism; - transposing the functional requirements into an appropriate algorithm (graphical, analytical), on the basis of which the kinematic synthesis is carried out; 
- obtaining the mechanism (meaning the specific geometric parameters);

- determining the kinematic functions achieved by the mechanism obtained from the synthesis process, and comparing their values with those imposed by the functional requirements, thus allowing to evaluate the deviations from the theoretical values;

- optimizing the mechanism for obtaining the appropriate functions, if the deviations obtained in the previous step are not convenient.

For the positional analysis of the multi-link mechanisms, vector-based methods (algebraic, matriceal) are frequently used for planar mechanisms [1]-[3]. In the case of spatial mechanisms, because of their complexity, the vector-based methods are difficult to apply, especially for the multi-loop (poly-contour) mechanisms, due to the large number of transformations would be required to express all the vectors attached to the elements in the base reference system.

In this situation, the geometric methods, mainly based on analytical constraint conditions, represent a good solution for the kinematic analysis of the spatial multi-link mechanisms [4]-[8]. The use of such methods is also justified by the need to define the mechanisms by the geometric parameters that are read directly from the execution drawings of the elements, and from the overall drawing of the system.

The scientific literature also presents mixed kinematic analysis algorithms, which combine geometric equations - to materialize the nature of the geometric constraints (joints) from the mechanism, and vectorial equations - by which the movement of the elements is modeled [9], [10].

On the other hand, powerful kinematic analysis algorithms are integrated in the commercial MBS (Multi-Body Systems) software environments, such as ADAMS of MSC.Software. Although these virtual prototyping tools provide important benefits, as pointed out in [11]-[15], their cost is still a major impediment, even in academic configurations.

In these terms, the current work proposes a numerical method for the kinematic analysis of the spatial multi-link mechanisms, which can be applied in unitary way to most types of linkages (especially the guiding linkages). As case study, the method is applied for a complex wheel guiding mechanism used for a vehicle suspension system, thus proving its usefulness (viability).

\section{Defining The Proposed Method}

The geometric model of the multi-link mechanisms is defined by the coordinates of some specific points on the mechanism rod (in the local technological frame of the rod) and on the base (in the global reference frame), as well by the lengths of the guiding links (bars) of the mechanism.

To explain the specifics of the method, the spatial multi-link mechanism shown in figure 2 is considered. The mechanism assures the guiding (in terms of spatial movement) of the central element / rod (2) by using three specific points, in this case the centers of the spherical joints $\mathrm{B}, \mathrm{C}$ and $\mathrm{D}$ to the adjacent elements $(1,3,4)$, which are guided on two circles (with the revolute axes A-A' and E-E') and one sphere (with the center in F). The constraints of the points through which the rod is guided in the spatial movement consist of the requirements that they be permanently on the support curves and /or surfaces, with centers on the mechanism base. The local reference frame of the $\operatorname{rod} \mathrm{X}_{2} \mathrm{Y}_{2} \mathrm{Z}_{2}$ has the origin in the rod center $\mathrm{O}, \mathrm{Y}_{2}$ is the longitudinal axis of the rod, $\mathrm{X}_{2}$ - the transversal axis, while $\mathrm{Z}_{2}$ is directed for obtaining a tri-orthogonal system.

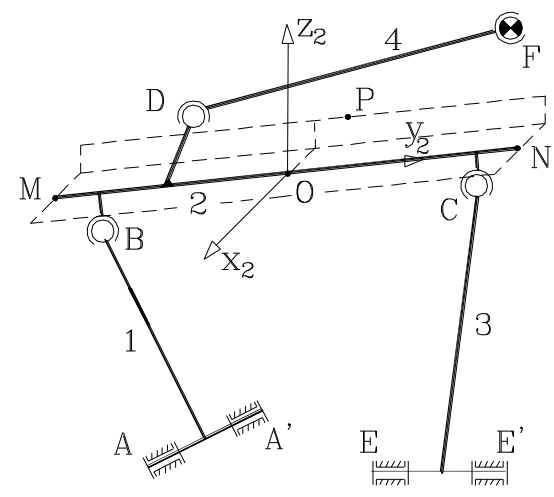

Fig. 2. The structural model of a spatial multi-link mechanism

The method proposed here is based on the premise that the spatial movement of the rod is completely defined by three non-collinear specific points, namely the two extremities of the $\operatorname{rod}(\mathrm{M}$ and $\mathrm{N})$, to which a third point $\mathrm{P}$ on the rod is added (see figure 2). The spatial positioning of the rod is defined by the set of positions of the three specific points, which determine the location and the orientation of the rod reference frame $\mathrm{X}_{2} \mathrm{Y}_{2} \mathrm{Z}_{2}$ relative to the global reference frame $\mathrm{XYZ}$ attached to the fixed base of the mechanism.

In the technological frame of the rod, the three specific points have the following local coordinates (which are input data for analysis): $\mathrm{M}\left(0, \mathrm{Y}_{\mathrm{M}(2)}, 0\right), \mathrm{N}\left(0, \mathrm{Y}_{\mathrm{N}(2)}, 0\right), \mathrm{P}\left(\mathrm{X}_{\mathrm{P}(2)}, \mathrm{Y}_{\mathrm{P}(2)}\right.$, $\mathrm{Z}_{\mathrm{P}(2)}$ ). In the global reference frame attached to the fixed base of the mechanism, the points have the global coordinates (which are to be determined through the kinematic analysis): $\mathrm{M}\left(\mathrm{X}_{\mathrm{M}}, \mathrm{Y}_{\mathrm{M}}, \mathrm{Z}_{\mathrm{M}}\right), \mathrm{N}\left(\mathrm{X}_{\mathrm{N}}, \mathrm{Y}_{\mathrm{N}}, \mathrm{Z}_{\mathrm{N}}\right), \mathrm{P}\left(\mathrm{X}_{\mathrm{P}}, \mathrm{Y}_{\mathrm{P}}, \mathrm{Z}_{\mathrm{P}}\right)$.

Between the three specific points, there can be defined three relationships, which are based on the corresponding constant distances equations (the rod is assumed to be rigid):

$$
\begin{aligned}
& F_{1}=\left(X_{P}-X_{N}\right)^{2}+\left(Y_{P}-Y_{N}\right)^{2}+\left(Z_{P}-Z_{N}\right)^{2}-|P N|^{2}=0, \\
& F_{2}=\left(X_{P}-X_{M}\right)^{2}+\left(Y_{P}-Y_{M}\right)^{2}+\left(Z_{P}-Z_{M}\right)^{2}-|P M|^{2}=0, \\
& F_{3}=\left(X_{N}-X_{M}\right)^{2}+\left(Y_{N}-Y_{M}\right)^{2}+\left(Z_{N}-Z_{M}\right)^{2}-|N M|^{2}=0,
\end{aligned}
$$

where the distances $|\mathrm{PN}|,|\mathrm{PM}|,|\mathrm{NM}|$ are computed in accordance with the local coordinates of the points (in the rod reference frame $\mathrm{X}_{2} \mathrm{Y}_{2} \mathrm{Z}_{2}$ ):

$$
\begin{aligned}
& |N M|=Y_{N(2)}-Y_{M(2)}, \\
& |P M|=\sqrt{X_{P(2)}^{2}+\left(Y_{P(2)}-Y_{M(2)}\right)^{2}+Z_{P(2)}^{2}}, \\
& |P N|=\sqrt{X_{P(2)}^{2}+\left(Y_{P(2)}-Y_{N(2)}\right)^{2}+Z_{P(2)}^{2}} .
\end{aligned}
$$

By considering as independent parameter (generalized coordinate) for the kinematic analysis one of the global coordinates of the specific points (one that is more 
appropriate, depending on the type of mechanism), to completely define the spatial positioning of the rod, besides equations (1), other five equations are still needed, and these equations are defined by considering the geometric constraints to which the rod is subjected (in correlation with the connections / joints to the adjacent elements of the mechanism). The following constraint equations correspond to the spatial multi-link mechanism shown in figure 2:

- for the guidance on sphere with center in $\mathrm{F}$ of the point $\mathrm{D}$ belonging to rod:

$$
F_{4}=\left(X_{D}-X_{F}\right)^{2}+\left(Y_{D}-Y_{F}\right)^{2}+\left(Z_{D}-Z_{F}\right)^{2}-l_{4}^{2}=0,
$$

- for the guidance on circle of the rod point $\mathrm{B}$, considering the revolute axis defined by the pair of points $\mathrm{A}-\mathrm{A}$ ':

$$
\begin{aligned}
& F_{5}=\left(X_{B}-X_{A}\right)^{2}+\left(Y_{B}-Y_{A}\right)^{2}+\left(Z_{B}-Z_{A}\right)^{2}-l_{1}^{2}=0 \\
& F_{6}=\left(X_{B}-X_{A^{\prime}}\right)^{2}+\left(Y_{B}-Y_{A^{\prime}}\right)^{2}+\left(Z_{B}-Z_{A^{\prime}}\right)^{2}-l_{1^{\prime}}^{2}=0
\end{aligned},
$$

- for the guidance on circle of the rod point $\mathrm{C}$, considering the revolute axis defined by the pair of points $\mathrm{E}-\mathrm{E}$ ':

$$
\begin{aligned}
& F_{7}=\left(X_{C}-X_{E}\right)^{2}+\left(Y_{C}-Y_{E}\right)^{2}+\left(Z_{C}-Z_{E}\right)^{2}-l_{3}^{2}=0 \\
& F_{8}=\left(X_{C}-X_{E^{\prime}}\right)^{2}+\left(Y_{C}-Y_{E^{\prime}}\right)^{2}+\left(Z_{C}-Z_{E^{\prime}}\right)^{2}-l_{3^{\prime}}^{2}=0
\end{aligned},
$$

The global coordinates of the points $\mathrm{A}-\mathrm{A}^{\prime}, \mathrm{E}-\mathrm{E}^{\prime}$ and $\mathrm{F}$ in the global reference frame are input data for the kinematic analysis (known parameters that define the geometric model of the mechanism).

In this way, a non-linear system of eight equations is obtained, the unknowns being the global coordinates of the three specific points $\mathrm{M}, \mathrm{N}$, and $\mathrm{P}$, excepting the independent coordinate, which is the imposed kinematic parameter. In the first three functions F1, F2, F3 (eq. 1), the eight unknowns appear explicitly, while in the other functions F4, ..., F8 (eq. 2 - 4) they appear implicitly through the coordinates of the rod joints (in this case, B, C, and D).

The relationship between the rod joints and the three specific points that define its position and orientation is defined by the conditions that the distances between each joint and the specific points are constant (the rod being a rigid body). As example, for the spherical joint $\mathrm{B}$, there are the following constant distance equations:

$$
\begin{aligned}
& T_{1}=\left(X_{B}-X_{M}\right)^{2}+\left(Y_{B}-Y_{M}\right)^{2}+\left(Z_{B}-Z_{M}\right)^{2}-|B M|^{2}=0, \\
& T_{2}=\left(X_{B}-X_{N}\right)^{2}+\left(Y_{B}-Y_{N}\right)^{2}+\left(Z_{B}-Z_{N}\right)^{2}-|B N|^{2}=0, \\
& T_{3}=\left(X_{B}-X_{P}\right)^{2}+\left(Y_{B}-Y_{P}\right)^{2}+\left(Z_{B}-Z_{P}\right)^{2}-|B P|^{2}=0,
\end{aligned}
$$

where the distances $|\mathrm{BM}|,|\mathrm{BN}|$ and $|\mathrm{BP}|$ are computed in correlation with the local coordinates of the points in the rod reference frame (which are known input data):

$$
\begin{aligned}
& |B M|=\sqrt{\left(X_{B(2)}\right)^{2}+\left(Y_{B(2)}-Y_{M(2)}\right)^{2}+\left(Z_{B}(2)\right)^{2}}, \\
& |B N|=\sqrt{\left(X_{B(2)}\right)^{2}+\left(Y_{B(2)}-Y_{N(2)}\right)^{2}+\left(Z_{B(2)}\right)^{2},} \\
& |B P|=\sqrt{\left(X_{B(2)}-X_{P(2)}\right)^{2}+\left(Y_{B}(2)-Y_{P(2)}\right)^{2}+\left(Z_{B(2))}-Z_{P(2)}\right)^{2} .}
\end{aligned}
$$

The non-linear system of three equations with three unknowns $\left(\mathrm{X}_{\mathrm{B}}, \mathrm{Y}_{\mathrm{B}}, \mathrm{Z}_{\mathrm{B}}\right)$ was solved by subtracting the first equation from the other two, resulting two linear equations in $X_{B}$ and $Y_{B}$ as functions of $Z_{B}$, as follows:

$$
X_{B}=a_{1} \cdot Z_{B}+b_{1}, Y_{B}=a_{2} \cdot Z_{B}+b_{2}
$$

These functions are replaced in the first equation of the system (5), resulting in this way a quadratic equation in $Z_{B}$ with the well-known solution

$$
Z_{B}=\frac{-b_{3} \pm \sqrt{b_{3}^{2}-a_{3} \cdot c_{3}}}{a_{3}} .
$$

The coefficients $a_{1,2,3}, b_{1,2,3}$ and $c_{3}$ depend on the global coordinates of the three specific points $(\mathrm{M}, \mathrm{N}, \mathrm{P})$ and the corresponding distances $(|\mathrm{BM}|,|\mathrm{BN}|,|\mathrm{BP}|)$. The coordinates $\mathrm{X}_{\mathrm{B}}$ and $\mathrm{Y}_{\mathrm{B}}$ are then obtained from eq. (6).

The non-linear system (5) is written for each joint of the rod (namely B, C and D for the multi-link mechanism shown in figure 2), thus resulting the coordinates for all of them, which will be then included / integrated in the constraint equations system of the mechanism F4, ..., F8 (eq. 2 - 4).

The global coordinates of the rod reference frame origin (the center of the rod axis $\mathrm{MN}$ ) are defined in the following way:

$$
X_{2}=\frac{X_{M}+X_{N}}{2}, Y_{2}=\frac{Y_{M}+Y_{N}}{2}, Z_{2}=\frac{Z_{M}+Z_{N}}{2} .
$$

The general non-linear system F1, ..., F8 (eq. 1 - 4) is solved by using the Newton-Kantorovich iterative method, starting from the following known initial solution:

$$
\begin{aligned}
& X_{M}=X_{2}^{0}, Y_{M}=Y_{2}^{0}+Y_{M(2)}, Z_{M}=Z_{2}^{0}, \\
& X_{N}=X_{2}^{0}, Y_{N}=Y_{2}^{0}+Y_{N(2)}, Z_{N}=Z_{2}^{0}, \\
& X_{P}=X_{2}^{0}+X_{P(2)}, Y_{P}=Y_{2}^{0}+Y_{P(2)}, Z_{P}=Z_{2}^{0}+Z_{P(2)} .
\end{aligned}
$$

The solving is performed in the following steps sequence: a. Establishing the initial solution (eq. 9) of the system.

b. Computing the coordinates of the rod reference frame origin (eq. 8), and the coordinates of the connection points (joints) of the rod (eq. 5), corresponding to the initial position of the mechanism;

c. Establishing the Jacobian of the system, by deriving the functions $F_{i}$ in relation with the unknown parameters $X_{i}$ :

$$
J=\left|\begin{array}{cccc}
\frac{v F_{1}}{v X_{1}} & \frac{v F_{1}}{v X_{2}} & \frac{v F_{1}}{v X_{3}} & \cdots \frac{v F_{1}}{v X_{i}} \\
\frac{v F_{2}}{v X_{1}} & \frac{v F_{2}}{v X_{2}} & \frac{v F_{2}}{v X_{3}} & \cdots \frac{v F_{2}}{v X_{i}} \\
\cdot & \cdot \\
\frac{v F_{i}}{v X_{1}} & \frac{v F_{i}}{v X_{2}} & \frac{v F_{i}}{v X_{3}} & \cdots \frac{v F_{i}}{v X_{i}}
\end{array}\right|, i=1 \ldots 8 .
$$


The partial derivatives of the first three functions $\mathrm{F}_{1}, \mathrm{~F}_{2}, \mathrm{~F}_{3}$ are directly obtained by deriving the rigid body conditions of the rod (eq. 1) in relation with the coordinates of the three specific points, which are explicitly found in these equations. For the other functions $\mathrm{F}_{4}-\mathrm{F}_{8}$, in which the unknowns are implicitly found, there are also considered the partial derivatives in relation with the coordinates of the rod joints, which are obtained by deriving the eq. (5).

d. Establishing the new solution of the system (in the first iteration) by using the Gauss-Jordan elimination, which is based on converting the corresponding matrix into reduced row echelon form.

e. testing the corresponding error:

$$
\left.\mid \begin{array}{c}
X_{P} \\
Y_{P} \\
Z_{P} \\
X_{M} \\
\vdots
\end{array}\right\rfloor_{1}-\left[\begin{array}{c}
X_{P} \\
Y_{P} \\
Z_{P} \\
X_{M} \\
\vdots
\end{array}\right\rfloor_{0} \leq|\varepsilon|,
$$

where $\varepsilon$ is the admissible error.

If expression (11) is satisfied, then ' 1 ' is retained as the solution of the system; otherwise, the iterative process is repeated from point/step ' $b$ ', assuming as the initial solution of the system in the new iteration the values of the unknowns from the previous iteration. The iterative process ' $a$ - $d$ ' ends when the difference between the values of the unknowns in two consecutive iterations ' $\mathrm{m}-1$ ' and ' $\mathrm{m}$ ' reaches the required precision:

$$
\left[\begin{array}{c}
X_{P} \\
Y_{P} \\
Z_{P} \\
X_{M} \\
\vdots
\end{array}\right]_{m}-\left[\begin{array}{c}
X_{P} \\
Y_{P} \\
Z_{P} \\
X_{M} \\
\vdots
\end{array}\right]_{m-1} \leq|\varepsilon|,
$$

the solution of the system being $\left\{X_{P}, Y_{P}, Z_{P}, X_{M}, Y_{M}, Z_{M}, X_{N}\right.$, $\left.\mathrm{Y}_{\mathrm{N}}, \mathrm{Z}_{\mathrm{N}}\right\}_{\mathrm{m}}$.

For a current position of the mechanism, the non-linear system is solved in similar way, by considering the solution obtained in the previous position of the mechanism as new initial solution. In this way, the kinematic behavior can be determined for the whole motion range (domain) of the mechanism.

\section{CASE STUdY}

The method proposed for the kinematic analysis of the spatial multi-link mechanisms was tested on various types of linkages. For this paper, the application was developed by considering the guiding linkage shown in figure 3 , which is frequently used in vehicle wheel suspension systems. In this case, the central element (i.e. the rod) of the mechanism is represented by the wheel carrier (6), which is guided in the relative motion to car body/chassis (the fixed base of the mechanism) by a set of five binary links (1-5), with spherical joints at both ends.

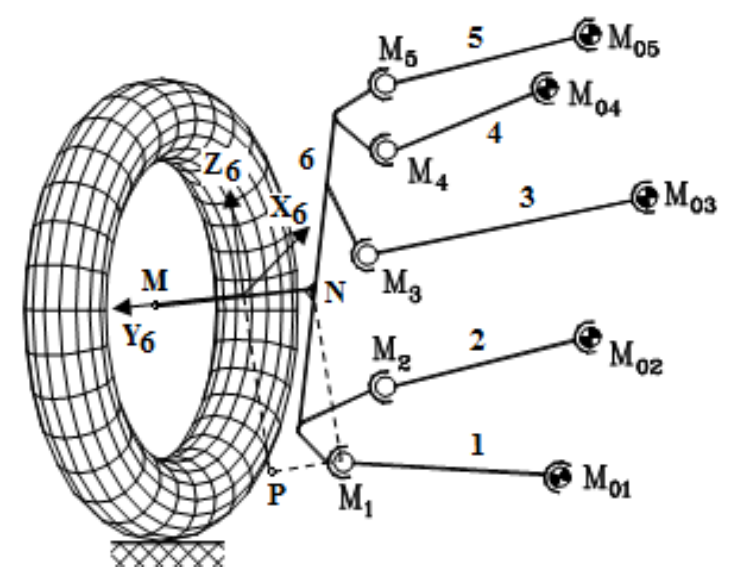

Fig. 3. The wheel guiding mechanism by five points - on five spheres (5S).

The five geometric constraints that describe the 5S-guiding mechanism are similar to that defined by eq. (2), as follows:

$$
F_{j}=\left(X_{M j}-X_{M 0 j}\right)^{2}+\left(Y_{M j}-Y_{M 0 j}\right)^{2}+\left(Z_{M j}-Z_{M 0 j}\right)^{2}-l_{j}^{2}=0,
$$

where $j=1 \ldots 5$ corresponds to the specific guiding links of the mechanism.

The three specific points that define the spatial position and orientation of the wheel carrier have been selected in the following way: the two extremities of the wheel carrier spindle ( $\mathrm{M}$ - the wheel center, and $\mathrm{N}$ ), the third point $(\mathrm{P})$ being located in the longitudinal - vertical plane of the wheel carrier reference frame $\mathrm{X}_{6} \mathrm{Y}_{6} \mathrm{Z}_{6}$.

The numerical algorithm for the kinematic analysis was transposed in computer code by using an object oriented programming language (Delphi). For this work, the kinematic study was performed by considering the passing over bumps regime, the independent kinematic parameter being the vertical coordinate of the wheel center $\mathrm{M}$, with the following motion domain $\mathrm{Z}_{\mathrm{M}} \in[-80,+80] \mathrm{mm}$.

Among the results obtained by running the program, figure 4.a shows the variation of the camber angle of the wheel, while figure 4.b shows several graphical simulation frames in the transversal - vertical plane YZ of the vehicle.

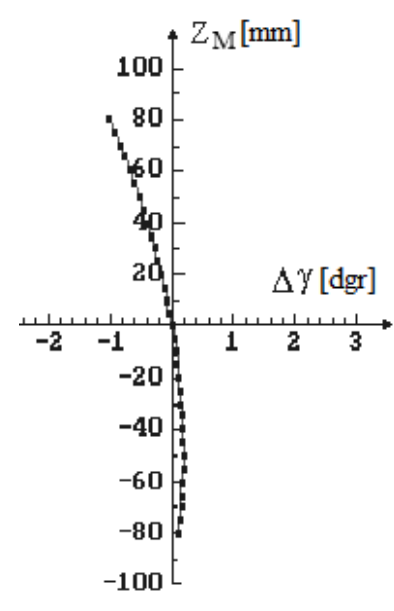

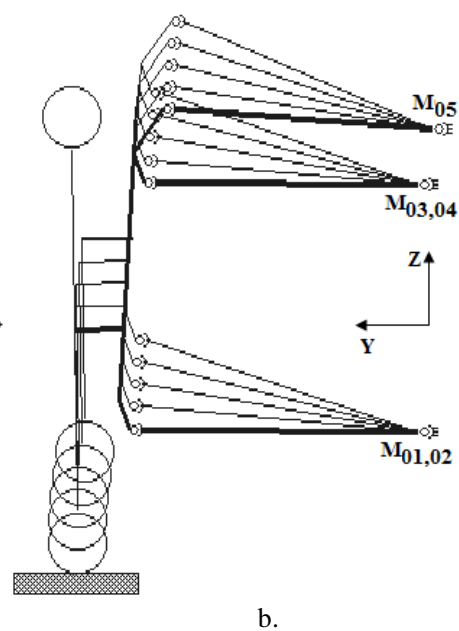

b.
Fig. 4. Results of the kinematic analysis for the 5S-guiding mechanism.

The camber angle represents the angle between the vertical axis of the wheel used for steering and the vertical axis of the 
vehicle, being analytically defined in the following way:

$$
\gamma=\operatorname{arctg} \frac{Z_{M}-Z_{N}}{Y_{M}-Y_{N}}, \Delta \gamma=\gamma-\gamma_{0},
$$

where $\gamma_{0}$ is the initial value of the camber angle, when the vehicle is in stationary position, corresponding to $\mathrm{Z}_{\mathrm{M}}=0$.

\section{CONCLUSIONS}

When designing a mechanism, after performing the synthesis, it is necessary to evaluate the behavior of the mechanism in order to identify the deviations from the imposed functional requirements. For the positional (kinematic) analysis of the spatial multi-link mechanisms, the paper proposes a general and unitary method, which can be applied to both mono-contour and poly-contour mechanisms. By appropriate customization, the method can be also applied to the planar multi-link mechanisms, in which case the position of the central element (i.e. the rod) of the mechanism is defined by only two specific points.

By reference to other methods from literature, the numerical algorithm proposed in this work brings several important benefits for the kinematic analysis of the multi-link mechanisms, such as the degree of applicability, the fast convergence of the non-linear system used to model the kinematic behavior of the mechanism, the accurate selection of the initial solution from which is going to solve the system.

The built in-house made computer program can be easily adapted to most of multi-link mechanism, the main advantages in relation to the commercial software products being the flexibility, and the greatly reduced costs.

\section{REFERENCES}

[1] C. Alexandru, Linkage Mechanisms (in Romanian), Transilvania University Press, 2000

[2] P. Alexandru, I. Vişa, C. Alexandru and D. Talabă, Functional Design of Mechanisms (in Romanian), Lux Libris, 1999.

[3] P. Alexandru, I. Vişa and C. Alexandru, "Modeling the angular capability of the ball joints in a complex mechanism with two degrees of mobility," Applied Mathematical Modelling, vol. 38, no. 23, pp. 5456-5470, 2014.

[4] C. Alexandru, "The kinematic optimization of the multi-link suspension mechanism used for rear axle of the motor vehicle," in Proc the Romanian Academy - Series A, vol. 10, no. 3, pp. 244-253, 2009.

[5] C. Alexandru, "Analytical algorithm for the optimal kinematic design of the wheel suspension mechanisms," Applied Mechanics and Materials, vol. 772, pp. 96-102, 2015.
[6] C. Alexandru and V. Țoţu, "Method for the multi-criteria optimization of car wheel suspension mechanisms," Ingeniería e Investigación, vol. 36, no. 2, pp. 60-67, 2016.

[7] H.A. Attia, "Kinematic analysis of the multi-link five-point suspension system in point coordinates," Journal of Mechanical Science and Technology, vol. 17, no. 8, pp. 1133-1139, 2003.

[8] J. Knapczyk and M. Maniowski, "Elastokinematic modeling and study of five-rod suspension with subframe," Mechanism and Machine Theory, vol. 41, no. 9, pp. 1031-1047, 2006.

[9] M. Hiller and C. Woernle, "Kinematical analysis of a five point wheel suspension," ATZ, vol. 87, no. 2, pp. 59-64, 1985.

[10] P.A. Simionescu and D. Beale, "Synthesis and analysis of the five-link rear suspension system used in automobile," Mechanism and Machine Theory, vol. 37, no. 9, pp. 815-832, 2002.

[11] C. Alexandru, "Software platform for analyzing and optimizing the mechanical systems," in Proc. the 10th IFToMM International Symposium on Science of Mechanisms and Machines - SYROM, pp. 665-677, Braşov, 2009.

[12] C. Alexandru, "Optimal design of the mechanical systems using parametric technique \& MBS (Multi-Body Systems) software,' Advanced Materials Research, vol. 463-464, pp. 1129-1132, 2012.

[13] M. Ceccarelli, "Challenges for mechanism design," in Proc. the 10th IFToMM International Symposium on Science of Mechanisms and Machines - SYROM, pp. 1-13, Braşov, 2009.

[14] D. Tarniţă and D. Marghitu, "Analysis of a hand arm system," Robotics and Computer-Integrated Manufacturing, vol. 29, pp. 493-501, 2013.

[15] D. Tarniță, M. Catană and D.N. Tarniță, "Contributions on the modeling and simulation of the human knee joint with applications to the robotic structures," Mechanisms and Machine Science, vol. 20, pp. 283-297, 2014.

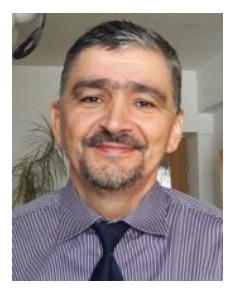

Cătălin Alexandru was born in Cetățeni, Romania, 1967. Bachelor (1991), PhD (1998), PhD supervisor (2009) in Mechanical Engineering at Transilvania University of Braşov, Romania.

He is currently professor at Transilvania University of Braşov, Romania, in the Product Design, Mechatronics and Environment Department. He published over 150 scientific articles, part of them in prestigious ISI-rated journals (such as Mechanism and Machine Theory, Applied Mathematical Modelling, Journal of Renewable and Sustainable Energy, The Scientific World Journal, Proceedings of the Institution of Mechanical Engineers - Part C: Journal of Mechanical Engineering Science). He owns 2 patents. He participated at numerous international conferences in Romania and abroad (England, Scotland, Portugal, Spain, France, Germany, Austria, Italy, and others). Research interests in mechanical \& mechatronic systems, automotive engineering, renewable energy systems, virtual prototyping.

Prof. Alexandru is member of International University Association for Science and Technology of Romania (UASTRO), and International Federation for the Promotion of Mechanism and Machine Science (IFToMM), editor for the Bulletin of the Transilvania University of Brasov: Series I - Engineering Sciences, and reviewer for prestigious ISI-rated journals (Advances in Mechanical Engineering, Applied Mathematical Modelling, Applied Mathematics and Computation, IEEE Transactions on Industrial Informatics, IEEE Transactions on Power Electronics, Journal of Mechanical Science and Technology, Journal of Renewable and Sustainable Energy, Mechanical Sciences, Mechanisms and Machine Theory, Robotics and Autonomous Systems, Solar Energy, Renewable Energy, and others). 